\title{
The GENDER ATTENTION Observational Study: Gender and Hormonal Status Differences in the Incidence of Adverse Events During Cyclosporine Treatment in Psoriatic Patients
}

\author{
Delia Colombo · Giuseppe Banfi • Nicoletta Cassano • Alessandra Graziottin • Gino Antonio Vena • \\ Giovanni Gualberto Fiori · Emanuela Zagni · Luca Stingeni · Sergio Chimenti • \\ Enzo Berardesca • Giuseppe Micali • Giuseppe Albertini • Clara De Simone • Gilberto Bellia • \\ On behalf of the GENDER ATTENTION study group
}

Received: January 27, 2017 / Published online: April 21, 2017

(c) The Author(s) 2017. This article is an open access publication

\section{ABSTRACT}

Introduction: Female sex has been shown to be a risk factor for the development of adverse drug reactions; however, this has not been studied

For a full list of the GENDER ATTENTION study group members please see the Acknowledgments.

Enhanced content To view enhanced content for this article go to http://www.medengine.com/Redeem/ 0C08F0604AA0168F.

D. Colombo $(\bowtie) \cdot$ E. Zagni

Novartis Farma S.p.A., Origgio, Varese, Italy

e-mail: delia.colombo@novartis.com

G. Banfi

IRCCS Galeazzi, Milan, Italy

G. Banfi

Università Vita e Salute San Raffaele, Milan, Italy

N. Cassano - G. A. Vena

University of Bari, Bari, Italy

A. Graziottin

Center of Gynecology and Medical Sexology, Milan, Italy

G. G. Fiori

Medineos Observational Research, Modena, Italy

L. Stingeni

Clinical, Allergological and Venereological

Dermatology Section, Department of Medicine,

University of Perugia, Perugia, Italy for cyclosporine (CsA). The aim of this study was to investigate, in Italian dermatological practice, the influence of gender and menopause and related hormones on the incidence of adverse events (AEs) during CsA treatment in psoriatic patients.

Methods: Multicenter, prospective, observational study conducted from May 2011 to June 2013. Patients with plaque psoriasis, undergoing a new CsA administration course, or about to start it, were enrolled in the outpatient clinics of Italian dermatological centers. During the

\section{S. Chimenti}

Dermatology, Tor Vergata University, Rome, Italy

\section{E. Berardesca}

San Gallicano Dermatology Institute, IRCCS, Rome, Italy

\section{G. Micali}

Department of Dermatology, University of Catania, Catania, Italy

\section{G. Albertini}

Dermatology, IRCCS Arcispedale Santa Maria

Nuova, Reggio Emilia, Italy

C. De Simone Institute of Dermatology, Università Cattolica del Sacro Cuore, Rome, Italy

\section{G. Bellia}

UCB Pharma Italia, Milan, Italy 
2-6 months of study duration, patients had to note all AEs that occurred in a diary that was reviewed by the investigators at the follow-up visit. Sex hormone levels were measured within 7 days from the start date of a menstrual cycle. Results: A total of 969 adult psoriatic patients were enrolled in the study, divided into four cohorts: fertile women and corresponding age-matched men; postmenopausal women and corresponding age-matched men. A significant difference in the percentage of patients with AEs was observed between fertile and postmenopausal women, but not between women and age-matched men. AE incidence rate was about $37 \%$ higher in fertile women than in age-matched men and about $18 \%$ higher in postmenopausal women than in age-matched men, but differences were not statistically significant. Incidence rate ratio of fertile vs. postmenopausal women was 0.67, reaching statistical significance. AEs were mild or moderate in severity in the great majority of patients of all cohorts and postmenopausal women had significantly less grade 1-2 AEs compared to fertile women, but more grade 3-4 AEs. FSH levels were significantly higher in postmenopausal women reporting no AEs, and DHEA sulfate levels were about 10\% higher in men with no AEs, compared to those reporting at least one AE. Cortisol levels were slightly though significantly higher in postmenopausal women with no AE.

Conclusions: A better understanding of sexand hormone-related influences on drug responses may help to improve drug safety and efficacy, by permitting one to tailor pharmacological treatments to individual subjects or defined patient cohorts.

Funding: Novartis Farma S.p.A., Italy.

Keywords: Adverse drug reaction; Cyclosporine; Dermatology; Female; Gender; Psoriasis

\section{INTRODUCTION}

Female sex has been shown to be a risk factor for the development of adverse drug reactions (ADRs) [1-3]. Adverse effects to a number of drugs appear to be more frequent and severe in women than in men $[4,5]$, and this is in line with the evidence that eight out of 10 drugs dropped out from the US market were responsible for more ADRs in women than in men [6]. The impact of pharmacodynamic and pharmacokinetic differences between sexes as well as of the physiological/hormonal status of women has been discussed. Furthermore, it has been pointed out that risk factors for ADRs, such as polytherapy, aging, and depression, are more frequent in women $[1-3,7,8]$.

Confounding factors biasing clinical trials results include small numbers of women, as well as weight or body mass index (BMI) and hormonal status not being considered [9]. Therefore, the US Food and Drug Administration (FDA) issued a guideline calling for the inclusion of women into early phases of clinical trials and the consideration of the effects of female physiology, such as hormones, during the study of drugs. Indeed, the optimization of therapy requires gender attention.

To our knowledge, no previous study analyzed the sex-specific incidence rate of AEs during CsA treatment of plaque psoriasis in real life. The GENDER ATTENTION study was designed with the aim of investigating, in Italian dermatological practice, the influence of gender and menopause on the incidence of AEs during cyclosporine (CsA) treatment. The study was conducted on a population of patients with plaque psoriasis, a disease that is rather equally distributed among genders [10].

\section{METHODS}

\section{Study Subjects}

Adult (age at least 18 years) male and female outpatients diagnosed with plaque psoriasis were enrolled, either naïve or previously treated with CsA, who had been undergoing a new CsA administration course for at most 30 days before, or about to start it within 14 days after study enrollment, with any therapeutic regimen according to the investigator's choice. Patients were divided into four cohorts of patients: fertile women (cohort 1) and age-matched men 
(cohort 2); women in menopause (cohort 3) and age-matched men (cohort 4). In order to evaluate differences between genders, men were planned to be matched to women in a $1: 2$ ratio into the following age groups: 18-34; 35-44; 45-54; 55+ years. Menopausal status was defined as the presence of amenorrhea for at least 12 months.

All subjects gave their informed consent for participation in the study. The study protocol was reviewed by the independent ethics committee (IEC) or institutional review board (IRB) of each center. The study was carried out in accordance with the approved applicable guidelines.

\section{Study Design}

This was a multicenter, prospective, observational, cohort study. After enrollment, patients were followed for a period of 2-6 months. Two study visits were planned: one enrollment visit and one follow-up visit at CsA discontinuation or at the end of follow-up or withdrawal from the study, whichever came first (min 2 months, max 6 months).

The primary objective was to evaluate the difference in adverse event (AE) incidence rate between fertile and postmenopausal women and age-matched men, during CsA treatment. Secondary objectives were to compare $\mathrm{AE}$ severity between genders, to describe AEs in relation to patient hormonal levels, and to assess overall patient satisfaction, as measured by the Treatment Satisfaction Questionnaire for Medication (TSQM) [11].

At the enrollment visit, sociodemographic data and medical history were collected, the Psoriasis Area and Severity Index (PASI) was evaluated, and the menopausal status was assessed in women on the basis of absence for at least the last 12 months of the menstrual cycle. Blood samples were drawn for hormonal level measurement: $\mathrm{E}_{2}, \mathrm{FSH}, \mathrm{LH}$, dehydroepiandrosterone (DHEA) sulfate, cortisol, testosterone; in fertile women blood had to be drawn within 7 days from the start date of the menstrual cycle. All enrolled patients were given a diary to note CsA treatment, concomitant medications, and AEs that occurred between baseline and the follow-up visit. At the follow-up visit, data on psoriasis treatment, other concomitant medications, and AEs were collected, and PASI was re-evaluated. The TSQM was administered at the follow-up visit.

As this was a non-interventional study, participants received CsA as per patients' needs and local clinical practice, and there were no constraints about concomitant treatment.

\section{Sample Size}

The number of available and affordable participants for this study was fixed at 1200 patients. We planned to enroll 400 fertile women and 200 age-matched men as well as 400 women in menopause and 200 age-matched men. Starting from the fixed sample size of 1200 enrolled patients, the minimum effect size that can be detected was computed. The information needed to calculate the minimum detectable incidence rate ratio (IRR), i.e., incidence rate of side effects in men and in fertile/postmenopausal women, was not available, since incidence rates have never been studied in the manner intended in this study. According to the study steering committee's opinion, on the basis of the Italian clinical experience with the doses used in dermatology that are largely beneath those considered at risk of AEs, an AE incidence rate of about 8 per 100 person-months was to be expected. Assuming that $20 \%$ of patients are not evaluable because of missing values or dropout, 960 subjects were expected to be included in the analyses: 320 fertile women and 160 age-matched men, and 320 women in menopause and 160 age-matched men. Assuming a type I error rate of 5\% with an allocation ratio of 1:2 (men vs. women) for each comparison, the minimum detectable IRR was computed as a function of the reference incidence rate (incidence rate from 4 to 16 side effects per 100 person-months) and of the average follow-up duration $(3,4$, and 5 months were considered). According to these assumptions, the minimum detectable IRR ranged from 1.24 (for an expected reference incidence rate of 16 side effects per 100 person-months and an average 
follow-up duration of 5 months) to 1.69 (for an expected reference incidence rate of 4 side effects per 100 person-months and an average follow-up duration of 3 months).

\section{Statistical Analysis}

The sample of patients included in the study was described in terms of sociodemographic and clinical characteristics at baseline, CsA treatment features, and concomitant medications. The proportion of patients experiencing at least one $\mathrm{AE}$ was computed in each cohort, overall and by event type. Differences among cohorts were assessed by means of Chi-squared tests. Grade of AEs was evaluated as well by comparing the number of grade $1-2$ vs. grade $>2$ events between patient cohorts (Chi-squared tests).

Treatment exposure was calculated as the number of months elapsed from the start to the end of the CsA treatment course or to the follow-up visit/withdrawal from the study. The incidence rate was calculated as the number of AEs occurred during the exposure period divided by the total person-time at risk of side effects. Comparisons of rates between fertile women vs. age-matched men, postmenopausal women vs. age-matched men, and fertile vs. postmenopausal women were performed by calculating IRRs and their 95\% confidence intervals.

IRRs of AEs between two cohorts were estimated using a Poisson regression model. The response variable was the count of AEs observed during the exposure period, while the covariate was the categorical variable that identified the cohort. The logarithm of person-time in months was included in the model with coefficient constrained to 1 (i.e., offset). In order to take into account data overdispersion, the scale parameter was estimated as the square root of the ratio between deviance and degrees of freedom. Moreover, multivariate Poisson regression models including potential confounder as covariate (one at a time) were provided.

Finally, hormone levels were described by occurrence of AEs in each cohort. Wilcoxon-Mann-Whitney tests were applied to evaluated differences between patients with and without AEs for some identified hormones.

\section{RESULTS}

Overall, 969 patients were enrolled into the study; 76 patients were excluded because of unavailability of the exposure period and four because they did not meet the inclusion criteria; 59 voluntarily withdrew or were lost to follow-up; 830 completed baseline evaluation (Fig. 1). During follow-up, one patient died for causes not related to CsA according to the investigator's judgment, and two withdrew for organizational reasons. Regarding male to female ratio, after a few months from enrollment start it was noticed that the 1:2 ratio was not achievable. This may be explained by the fact that women are less willing to participate in studies than men for reasons of time availability. Thus, male cohorts were increased in order to maximize the number of patients included in the analyses. In each age class (18-34, 35-44, 45-54, 55+), the proportion of fertile and postmenopausal women was computed and an equal proportion of randomly selected men was matched to each group (Table 1). Finally, the safety analysis included 336 fertile women and 253 age-matched men as well as 182 postmenopausal women and 118 age-matched men. Sociodemographic and baseline features of each study cohort are summarized in Table 1. Participants were $97.4 \%$ Caucasian with no differences between cohorts. In cohort 3 , the median time since menopause was 66 months. At study entry, $28.6 \%$ of patients showed at least one concomitant disease. That proportion was higher in the older cohorts, but no significant differences were observed between women and age-matched men cohorts $(22.3 \%$ in cohort $1,19.0 \%$ in cohort $2,46.2 \%$ in cohort 3 , and $39.8 \%$ in cohort 4). The most common concomitant disease at baseline in all cohorts was hypertension: $3.3 \%$ and $5.1 \%$ in cohorts 1 and 2, respectively, and $21.4 \%$ and $16.9 \%$ in cohorts 3 and 4, respectively. In the enrolled sample no patient was naïve to CSA treatment. A treatment course of 7 days a week for about 


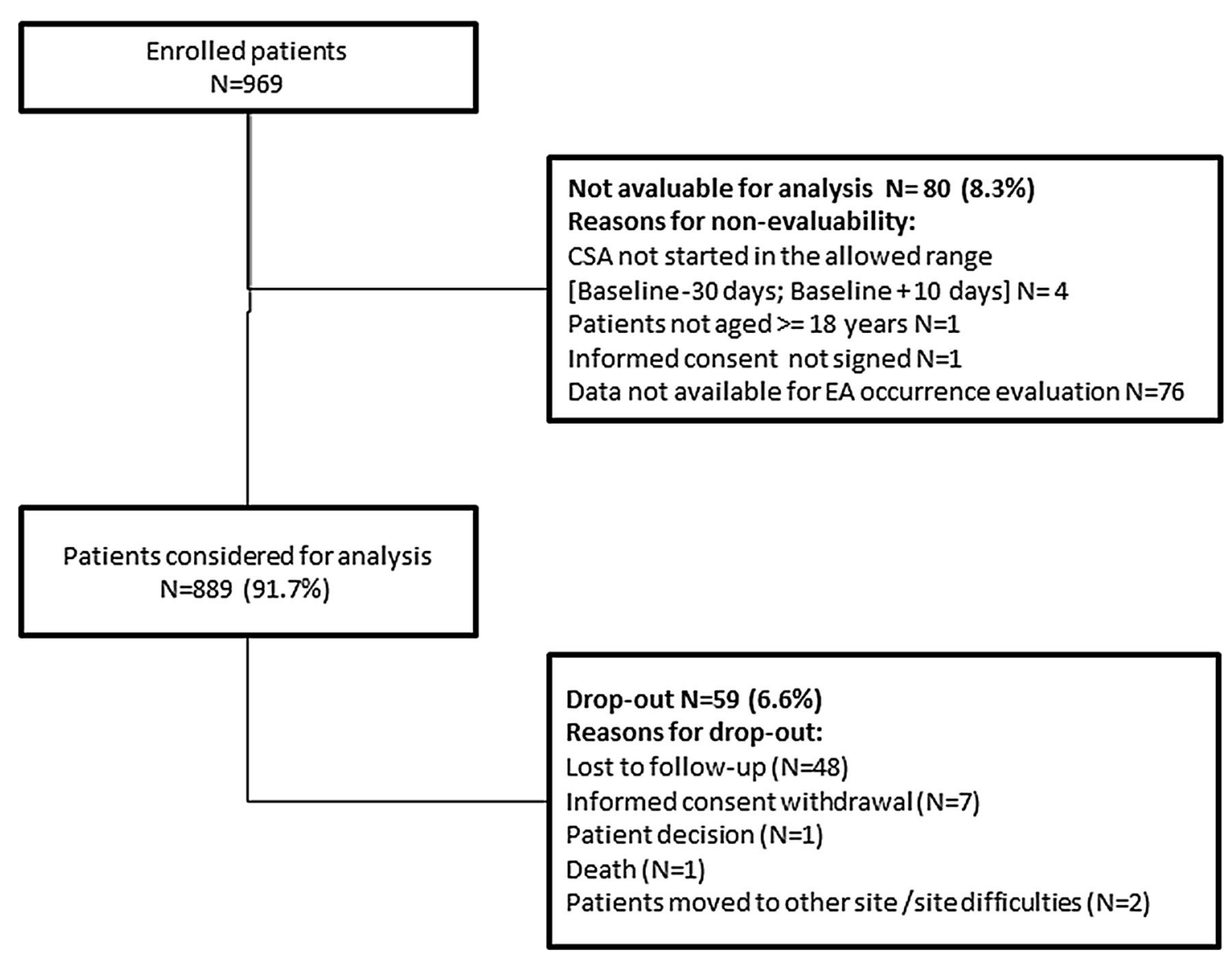

Fig. 1 Patient disposition. The figure shows the number of patients considered for the analysis and who dropped out. Reasons for non-evaluability or dropout could be multiple

17-20 weeks was the most used CsA treatment regimen. During this period, patients received up to three doses of cyclosporine every day (11.6\% one, $79.0 \%$ two, and the remaining three doses). The average daily dose (SD) of cyclosporine was $3.3(0.8) \mathrm{mg} / \mathrm{kg}$ in cohort 1 , $3.2(0.8) \mathrm{mg} / \mathrm{kg}$ in cohort $2,3.1(0.7) \mathrm{mg} / \mathrm{kg}$ in cohort 3 , and $3.0(0.9) \mathrm{mg} / \mathrm{kg}$ in cohort 4 . Topical therapies were the most common concomitant treatment in all cohorts with a percentage ranging from $28.6 \%$ in cohort 3 to $41.5 \%$ in cohort 4 . Among other treatments for psoriasis, $1.7 \%$ of patients received phototherapy and $4.4 \%$ systemic therapies. Concomitant hormonal treatments were used almost exclusively in cohort 1 , with $9.5 \%$ of fertile women taking the contraceptive pill, and a small percentage receiving hormone replacement therapy $(1.8 \%)$.
Overall, 302 patients reported at least one AE (34.0\%). The percentage of patients with AEs was $31.3 \%$ in cohort $1,29.6 \%$ in cohort 2 , $42.3 \%$ in cohort 3 , and $38.1 \%$ in cohort 4 (Fig. 2). Incidence and type of AEs are reported in Table 2. A significant difference was observed between fertile and postmenopausal women (Chi-squared $p$ value 0.0118 ), but no statistically significant differences were observed in fertile and postmenopausal women compared to age-matched men (Chi-squared $p$ value 0.675 and 0.472 , respectively). The IRR of fertile women compared to age-matched men was 1.37 (95\% CI 1.00-1.86), while IRR comparing postmenopausal women vs. age-matched men was 1.18 (95\% CI 0.78-1.79). Thus, AE incidence rate was about $37 \%$ higher in fertile women than in age-matched men and about 18\% higher in postmenopausal women than in 
Table 1 Sociodemographic and disease baseline features

\begin{tabular}{|c|c|c|c|c|}
\hline Variable & $\begin{array}{l}\text { Cohort 1: } \\
\text { fertile } \\
\text { women }\end{array}$ & $\begin{array}{l}\text { Cohort 2: age-matched } \\
\text { men (to fertile women) }\end{array}$ & $\begin{array}{l}\text { Cohort 3: } \\
\text { postmenopausal } \\
\text { women }\end{array}$ & $\begin{array}{l}\text { Cohort 4: age-matched men } \\
\text { (to postmenopausal women) }\end{array}$ \\
\hline$N$ & 336 & 253 & 182 & 118 \\
\hline \multirow[t]{2}{*}{ Age (years), mean (SD) } & $35.56(9.03)$ & $(p=0.039)^{\S}$ & $57.13(5.68)$ & $(p=0.011)^{\#}$ \\
\hline & & $37.06(8.78)$ & & $55.46(6.07)$ \\
\hline \multicolumn{5}{|l|}{ Age group, $n$} \\
\hline $18-34$ & 151 & 94 & 0 & 0 \\
\hline $35-44$ & 124 & 110 & 1 & 1 \\
\hline $45-54$ & 60 & 48 & 58 & 46 \\
\hline $55+$ & 1 & 1 & 123 & 71 \\
\hline Education, $n(\%)^{*}$ & & $(p=0.099)^{\S}$ & & $(p=0.234)^{\#}$ \\
\hline None & $0(0.0)$ & $0(0.0)$ & $2(1.1)$ & $1(0.8)$ \\
\hline Primary school & $8(2.4)$ & $10(4.0)$ & $35(19.2)$ & $15(12.7)$ \\
\hline Lower secondary & $90(26.8)$ & $79(31.2)$ & $62(34.1)$ & $51(43.2)$ \\
\hline Upper secondary & $175(52.1)$ & $133(52.6)$ & $60(33.0)$ & $40(33.9)$ \\
\hline Bachelor degree & $54(16.1)$ & $25(9.9)$ & $18(9.9)$ & $8(6.8)$ \\
\hline \multirow[t]{2}{*}{ Caucasian race, $N(\%)$} & $328(97.6 \%)$ & $(p=0.395)^{\S}$ & $178(97.8 \%)$ & $(p=0.453)^{\#}$ \\
\hline & & $243(96.0 \%)$ & & $117(99.2 \%)$ \\
\hline \multirow{2}{*}{$\begin{array}{l}\text { Age at diagnosis (years), } \\
\text { mean (SD) }\end{array}$} & \multirow{2}{*}{$\begin{array}{l}23.81 \\
\quad(10.59)\end{array}$} & $(p=0.371)^{\S}$ & $40.85(13.61)$ & $(p=0.813)^{\#}$ \\
\hline & & $24.87(10.18)$ & & $40.74(13.01)$ \\
\hline \multirow{2}{*}{$\begin{array}{l}\text { Disease duration (years), } \\
\text { mean (SD) }\end{array}$} & \multirow{2}{*}{$\begin{array}{l}12.13 \\
(10.08)\end{array}$} & $(p=0.366)^{\S}$ & \multirow[t]{2}{*}{$16.75(12.97)$} & $(p=0.120)^{\#}$ \\
\hline & & $12.58(9.11)$ & & $15.12(10.82)$ \\
\hline \multicolumn{2}{|l|}{$\begin{array}{l}\text { PASI at enrollment, } \\
n(\%)^{*}\end{array}$} & $(p=0.836)^{\S}$ & & $(p=0.272)^{\#}$ \\
\hline$\leq 20$ & $258(84.6)$ & $192(83.5)$ & $132(81.5)$ & $90(80.4)$ \\
\hline $21-29$ & $35(11.5)$ & $30(13.0)$ & $22(13.6)$ & $20(17.9)$ \\
\hline$\geq 30$ & $12(3.9)$ & $8(3.5)$ & $8(4.9)$ & $2(1.8)$ \\
\hline \multirow{2}{*}{$\begin{array}{l}\text { Months from CSA start, } \\
\text { mean (SD) }\end{array}$} & \multirow{2}{*}{$\begin{array}{l}40.76 \\
\quad(42.19)\end{array}$} & $(p=0.772)^{\S}$ & $48.39(52.60)$ & $(p=0.140)^{\#}$ \\
\hline & & $41.58(39.97)$ & & $37.74(47.02)$ \\
\hline \multirow{2}{*}{$\begin{array}{l}\text { No. of previous CSA } \\
\text { cycles, mean (SD; } \\
\text { min-max) }\end{array}$} & \multirow{2}{*}{$\begin{array}{c}2.09(1.71 \\
1-10)\end{array}$} & $(p=0.684)^{\S}$ & \multirow[t]{2}{*}{$1.98(1.44 ; 1-7)$} & $(p=0.0684)^{\#}$ \\
\hline & & $2.19(2.07 ; 1-14)$ & & $2.60(2.08 ; 1-10)$ \\
\hline
\end{tabular}


Table 1 continued

\begin{tabular}{lllll}
\hline Variable & $\begin{array}{l}\text { Cohort 1: } \\
\text { fertile } \\
\text { women }\end{array}$ & $\begin{array}{l}\text { Cohort 2: age-matched } \\
\text { men (to fertile women) }\end{array}$ & $\begin{array}{l}\text { Cohort 3: } \\
\text { postmenopausal } \\
\text { women }\end{array}$ & $\begin{array}{l}\text { Cohort 4: age-matched men } \\
\text { (to postmenopausal women) }\end{array}$ \\
\hline $\begin{array}{l}\text { No. of days of CSA } \\
\text { treatment, mean (SD) }\end{array}$ & $\begin{array}{c}130.00 \\
(54.13)\end{array}$ & $\begin{array}{l}(p=0.009)^{\S} \\
141.40(51.28)\end{array}$ & $122.59(54.62)$ & $(p=0.007)^{\#}$ \\
\hline
\end{tabular}

The $t$ test for equal variances was considered for continuous variables, Chi-squared test for categorical variables

* Percentages are computed on the basis on patients with available data

$\S$ Fertile women vs. age-matched men (to fertile women)

\# Postmenopausal women vs. age-matched men (to postmenopausal women)

age-matched men, but no significant differences between female and male cohorts were observed. IRR of fertile vs. postmenopausal women was 0.67 (95\% CI 0.49-0.92), reaching statistical significance. Severity of AEs, according to the Common Technology Criteria for Adverse Events (CTCAE), is summarized in Table 3. AEs were mild (grade 1, 47.6-60.2\%) or moderate (grade 2, 35.2-40.1\%) in the great majority of patients of all cohorts. However, a significant difference between severity of the $\mathrm{AE}$ (grade 1-2 vs. $>2$ ) and patient's cohort was observed, with postmenopausal women having less low grade AEs and more grade 3-4 AEs compared to fertile women $(87.7 \%$ vs. $95.5 \%$ of AEs experienced by postmenopausal and fertile women were grade $1-2$, respectively, while $12.3 \%$ vs. $4.5 \%$ were grade $3-4, p=0.0005$ ). Considering individual AEs, hypertension was significantly less reported in both cohort 1 and 2 (younger females and males) compared to cohort 3 and 4 (older females and males) respectively $(p=0.002$ and $p=0.02$, respectively). Once adjusted for age, education level, BMI, psoriasis duration, presence of concomitant diseases, presence of concomitant treatments, and baseline PASI (considered one at a time), IRR comparing fertile women versus age-matched men did not change (min 1.31, max 1.38), while adjusting for time elapsed from the start of CsA treatment, IRR decreased to 1.09. Similarly, comparing women in menopause versus age-matched men, IRR did not change adjusting for age, education level, BMI, presence of concomitant diseases, and presence of concomitant treatments (considered one at a time) (min 1.17, $\max 1.23$ ), while an increase, though not statistically significant, was obtained adjusting for other potential confounders, such as duration of psoriasis (1.35), time elapsed from CsA treatment start (1.37), and PASI (1.41). Anyway, variations in IRR due to controlling for potential confounders seem to be negligible.

Hormone levels stratified by patients with and without at least one $\mathrm{AE}$ in the four cohorts are reported in Table 4. Hormone levels in postmenopausal women confirmed their menopausal status. As expected, in postmenopausal women $\mathrm{E}_{2}$ levels were reduced to men's levels, while conversely FSH and LH levels were significantly higher than in fertile women. Median FSH levels were significantly higher in postmenopausal women reporting no AEs compared to those with at least one $\mathrm{AE}$ (Wilcoxon-Mann-Whitney test $p=0.0284$ ). DHEA sulfate levels were consistently lower in postmenopausal women than in fertile women and in men, but do not seem to correlate with occurrence of AEs. Among men, DHEA sulfate levels were about $10 \%$ higher in those with no AEs vs. those with AEs. Cortisol levels were rather homogeneous among cohorts, but were slightly significantly higher in postmenopausal women with no AE (Wilcoxon-Mann-Whitney test $p=0.0274)$. Median testosterone levels were a little higher (NS) in both postmenopausal women and men with no $\mathrm{AE}$ compared to those reporting AEs. Overall treatment satisfaction, measured by TSQM, was 


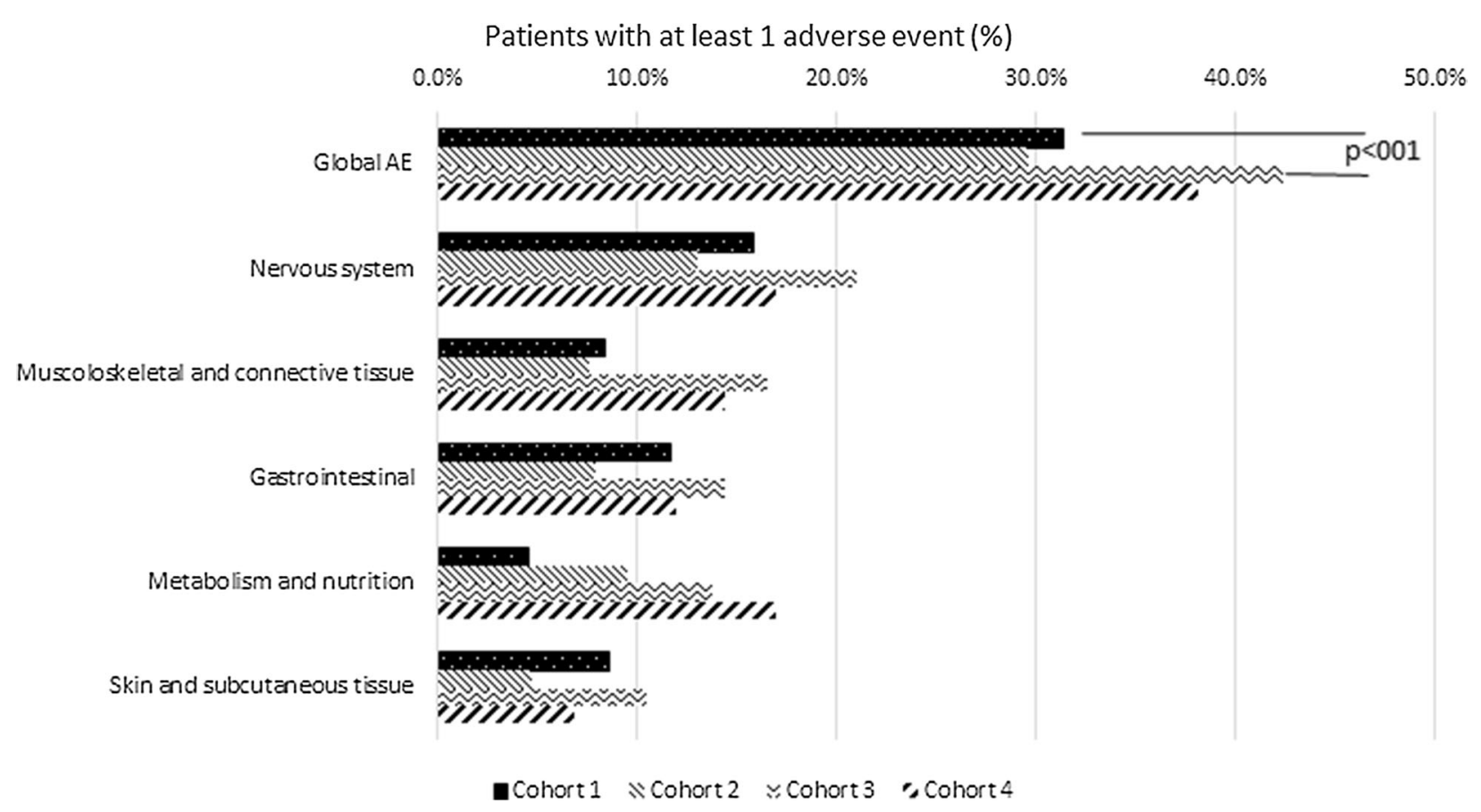

Fig. 2 Proportion of patients with at least one adverse event by patient cohort, overall, and by system organ class (the most frequent ones are displayed). For each cohort, proportions are computed as the ratio between the number of patients experiencing at least one adverse event during the exposure period and the total number of evaluable patients. The number of evaluable patients was 336 for

rather high (mean score from 67 to 91 for all items considering all patients) with no differences between cohorts, except for a slightly lower side effects score in postmenopausal women, consistent with the higher AE incidence rate in this cohort.

\section{DISCUSSION}

To our knowledge, this is the first large clinical study specifically designed to assess potential gender- and hormone-related differences in AEs associated with CsA treatment. Since CsA dosage is based on weight, we considered it a "clean" drug model to analyze the real difference between genders [12]. Psoriasis was chosen as disease model because it is usually treated with one systemic drug at a time only. As this was a non-interventional study, participants fertile women (cohort 1), 253 for age-matched men (cohort 2), 182 for postmenopausal women (cohort 3), and 118 for age-matched men (cohort 4). The $p$ value of the Chi-squared test between female cohort (fertile/postmenopausal) and experience of adverse events (yes/no) is shown as well

received CsA as per individual needs and local clinical practice, and there were no constraints about concomitant treatment.

Our results show a slightly higher IRR of AEs in fertile women compared to age-matched men $(+37 \%)$ and in postmenopausal women compared to age-matched men $(+18 \%)$, though both differences failed to show statistical significance. These findings are confirmed even when adjusted for potential confounders. Several published data report an association between female sex and frequency of ADRs [13-22], which has been hypothesized to be due to the higher prevalence of headache, migraine, and musculoskeletal pain in women, the higher prevalence of women among the elderly population, and the greater attention women pay to their health status. Moreover, menstrual cycles, pregnancy, and menopause are likely to have a relevant impact on pharmacokinetics and 
Table 2 Incidence of adverse events by preferred term $(\geq 1 \%$ incidence in any cohort)

\begin{tabular}{|c|c|c|c|c|}
\hline Patients with AE, $n$ (\%) & Cohort 1 & Cohort 2 & Cohort 3 & Cohort 4 \\
\hline Headache & $38(11.3)$ & $20(7.9)$ & $21(11.5)$ & $13(11.0)$ \\
\hline Hypertension & $12(3.6)$ & $19(7.5)$ & $19(10.4)$ & $18(15.3)$ \\
\hline Fatigue & $14(4.2)$ & $14(5.5)$ & $12(6.6)$ & $4(3.4)$ \\
\hline Muscle cramps & $11(3.3)$ & $7(2.8)$ & $15(8.2)$ & $8(6.8)$ \\
\hline Nausea & $18(5.4)$ & $7(2.8)$ & $8(4.4)$ & $6(5.1)$ \\
\hline Abdominal pain & $14(4.2)$ & $6(2.4)$ & $11(6.0)$ & $7(5.9)$ \\
\hline Muscle weakness & $13(3.9)$ & $6(2.4)$ & $9(4.9)$ & $7(5.9)$ \\
\hline Paresthesia & $12(3.6)$ & $9(3.6)$ & $5(2.7)$ & $6(5.1)$ \\
\hline Myalgia & $9(2.7)$ & $5(2.0)$ & $11(6.0)$ & $3(2.5)$ \\
\hline Diarrhea & $12(3.6)$ & $2(0.8)$ & $8(4.4)$ & $5(4.2)$ \\
\hline Tremor & $9(2.7)$ & $2(0.8)$ & $10(5.5)$ & $4(3.4)$ \\
\hline Gingival hyperplasia & $10(3.0)$ & $5(2.0)$ & $4(2.2)$ & $5(4.2)$ \\
\hline Hypertrichosis & $10(3.0)$ & $4(1.6)$ & $8(4.4)$ & $2(1.7)$ \\
\hline Insomnia & $4(1.2)$ & $8(3.2)$ & $6(3.3)$ & $2(1.7)$ \\
\hline Visual disturbances & $5(1.5)$ & $2(0.8)$ & $7(3.8)$ & $4(3.4)$ \\
\hline Agitation & $7(2.1)$ & $2(0.8)$ & $5(2.7)$ & 0 \\
\hline Vomit & $3(0.9)$ & $2(0.8)$ & $5(2.7)$ & $1(0.8)$ \\
\hline Weight gain & $3(0.9)$ & $2(0.8)$ & $6(3.3)$ & 0 \\
\hline Hyperlipidemia & $1(0.3)$ & $5(2.0)$ & $2(1.1)$ & $2(1.7)$ \\
\hline Menstrual disorders & $8(2.4)$ & - & $1(0.5)$ & - \\
\hline Vascular events & $3(0.9)$ & 0 & $3(1.6)$ & $1(0.8)$ \\
\hline Arthralgia & $2(0.6)$ & $2(0.8)$ & $2(1.1)$ & $1(0.8)$ \\
\hline Renal dysfunction & $1(0.3)$ & $3(1.2)$ & 0 & $2(1.7)$ \\
\hline Confusion & $2(0.6)$ & $1(0.4)$ & $2(1.1)$ & 0 \\
\hline Disorientation & $1(0.3)$ & 0 & $3(1.6)$ & 0 \\
\hline Edema & $1(0.3)$ & $1(0.4)$ & $2(1.1)$ & 0 \\
\hline Hepatic laboratory alterations & 0 & $1(0.4)$ & $2(1.1)$ & 0 \\
\hline Swollen limbs & 0 & 0 & $1(0.5)$ & $2(1.7)$ \\
\hline
\end{tabular}

pharmacodynamic of drugs [23]. Several sex-related factors, including hormonal status and genetics, have been proposed to explain why women experience more ADRs in response to viral vaccines and antiviral drugs [24].
However, in our study, we found only a small non-significant difference between genders, which may be explained by the fact that CsA, unlike the majority of current drugs, is given on a body weight basis, which reduces one major 
Table 3 Severity of adverse events (AEs) according to CTCAE v. 3.0

\begin{tabular}{lllll}
\hline Severity of AEs & $\begin{array}{l}\text { Cohort } \mathbf{1} \\
\boldsymbol{n}(\%)\end{array}$ & $\begin{array}{l}\text { Cohort 2 } \\
\boldsymbol{n}(\%)\end{array}$ & $\begin{array}{l}\text { Cohort 3 } \\
\boldsymbol{n}(\%)\end{array}$ & $\begin{array}{l}\text { Cohort 4 } \\
\boldsymbol{n}(\%)\end{array}$ \\
\hline 1 & $200(60.2)$ & $113(56.8)$ & $120(47.6)$ & $88(55.7)$ \\
2 & $117(35.2)$ & $70(35.2)$ & $101(40.1)$ & $60(38.0)$ \\
3 & $15(4.5)$ & $15(7.5)$ & $28(11.1)$ & $9(5.7)$ \\
4 & 0 & $1(0.5)$ & $3(1.2)$ & 0 \\
5 & 0 & 0 & 0 & $1(0.6)$ \\
\hline
\end{tabular}

Chi-squared test between severity of adverse events (CTCAE v. 3.0 grade 1-2/>2) and fertile/postmenopausal women, $p=0.0005$

Chi-squared test between severity of adverse events and fertile women/matched men and postmenopausal women/matched men, respectively, not significant

Percentages are computed from the number of adverse events that occurred in each patient cohort

$C T C A E$ common technology criteria for adverse events

gender impact. Actually, women are generally treated with doses that essentially reflect the results obtained by trials carried out mainly in men, and this has been hypothesized as one important reason for the higher incidence of drug adverse effects observed in women. Pharmaceutical companies should pay more attention, from the early drug development phases, to find out gender-appropriate doses.

On the other hand, in our study a significant difference between fertile and postmenopausal women emerged, which suggests that hormonal status and age may affect CsA tolerability. Also differences between men and women appear to be higher when hormonal differences exist, i.e., in the fertile age. Menopause is associated with changes not only in hormonal status but also in inflammatory factors, including cytokines, that have shown an impact on drug response [24, 25]. It can be hypothesized that menopause might thus have some influence also on drug tolerability. Higher cortisol and testosterone levels in postmenopausal women with no AEs might suggest a potential slightly protective effect of these hormones (Table 4; Fig. 3). No other clinically relevant associations were found between hormone levels and incidence of AEs. During menopause FSH levels are normally high; in fact, FSH levels support the diagnosis of menopause: this was also observed in our sample. We do not expect that FSH could have played any protective role within postmenopausal women.
A previous gender analysis in psoriatic patients, the PSYCHAE study, showed that Italian dermatologists seem to have the same approach in treating male and female patients with psoriasis, although women might warrant more attention to their psychological distress, which was higher than in men [26]. The GENDER ATTENTION study further suggests that greater attention in treating psoriatic patients with CsA should be paid to older patients and postmenopausal women.

Although this was not a study objective, it is worth mentioning that our results show a reassuring safety and tolerability profile of CsA, at the doses used to treat psoriasis in Italian common dermatological practice, with the great majority of patients of all cohorts having AEs of low or moderate severity. The most commonly occurring AE was headache, which showed higher incidence than expected from the known CsA tolerability profile, but it has to be considered that this type of event is quite common and can occur for lots of reasons. Among the most common CsA side effects [12], only hypertension was rather common in all our cohorts, significantly more so in older cohorts of both genders, this being explained by the fact that blood pressure tends to increase with age and prevalence of hypertension is higher among aged people. Hypertension is also expected according to previous literature [12]. The low overall proportion of patients with 
Table 4 Hormone levels in the different study populations, stratified by presence or absence of adverse effects (AEs)

\begin{tabular}{|c|c|c|c|}
\hline Hormone & With no AEs & With $\geq 1$ AEs & $p$ value \\
\hline \multicolumn{4}{|l|}{ Fertile women } \\
\hline 17-beta-estradiol $(\mathrm{pg} / \mathrm{mL})$ & $32.71(21.84 ; 54.92)$ & 33.17 (19.60; 49.78) & \\
\hline FSH $(\mathrm{mIU} / \mathrm{mL})$ & $6.68(4.55 ; 9.59)$ & $6.83(4.71 ; 10.74)$ & \\
\hline $\mathrm{LH}(\mathrm{mIU} / \mathrm{mL})$ & $9.36(6.22 ; 12.91)$ & $8.69(5.11 ; 12.56)$ & \\
\hline DHEA sulfate $(\mathrm{mg} / \mathrm{mL})$ & $147.00(94.00 ; 228.00)$ & $157.50(108.00 ; 256.50)$ & 0.2683 \\
\hline Cortisol (mg/dL) & $12.05(8.32 ; 17.61)$ & $11.18(7.85 ; 15.34)$ & \\
\hline Testosterone (ng/dL) & $51.00(34.00 ; 68.00)$ & $55.50(44.00 ; 68.00)$ & \\
\hline Free testosterone $(\mathrm{pg} / \mathrm{mL})$ & $1.06(0.46 ; 2.20)$ & $1.10(0.56 ; 2.55)$ & \\
\hline \multicolumn{4}{|l|}{ Postmenopausal women } \\
\hline 17-beta-estradiol $(\mathrm{pg} / \mathrm{mL})$ & $16.39(11.06 ; 27.67)$ & $14.99(10.81 ; 31.18)$ & \\
\hline $\mathrm{FSH}(\mathrm{mIU} / \mathrm{mL})$ & $60.59(45.67 ; 91.05)$ & $54.01(38.70 ; 79.76)$ & 0.0284 \\
\hline $\mathrm{LH}(\mathrm{mIU} / \mathrm{mL})$ & $42.05(33.11 ; 53.11)$ & $42.51(30.41 ; 52.72)$ & \\
\hline DHEA sulfate $(\mathrm{mg} / \mathrm{mL})$ & $83.00(47.00 ; 120.00)$ & $71.00(43.00 ; 126.00)$ & 0.4575 \\
\hline Cortisol (mg/dL) & $12.85(9.49 ; 17.02)$ & $10.69(7.41 ; 14.96)$ & 0.0274 \\
\hline Testosterone $(\mathrm{ng} / \mathrm{dL})$ & $44.50(31.00 ; 61.50)$ & $42.00(28.00 ; 57.00)$ & 0.1755 \\
\hline Free testosterone $(\mathrm{pg} / \mathrm{mL})$ & $0.78(0.37 ; 1.52)$ & $0.51(0.13 ; 1.12)$ & \\
\hline \multicolumn{4}{|l|}{ Men } \\
\hline 17-beta-estradiol (pg/mL) & $19.86(13.65 ; 30.06)$ & $19.26(14.89 ; 34.23)$ & \\
\hline $\mathrm{FSH}(\mathrm{mIU} / \mathrm{mL})$ & $4.22(2.98 ; 6.49)$ & $4.87(3.32 ; 6.36)$ & \\
\hline $\mathrm{LH}(\mathrm{mIU} / \mathrm{mL})$ & $7.67(5.48 ; 10.20)$ & $6.99(5.36 ; 10.00)$ & \\
\hline DHEA sulfate $(\mathrm{mg} / \mathrm{mL})$ & $197.00(123.00 ; 341.00)$ & $181.00(116.00 ; 277.00)$ & \\
\hline Cortisol (mg/dL) & $11.42(8.73 ; 15.72)$ & $11.25(8.44 ; 15.62)$ & \\
\hline Testosterone (ng/dL) & $404.00(319.00 ; 513.00)$ & $387.00(288.00 ; 463.00)$ & 0.0827 \\
\hline Free testosterone $(\mathrm{pg} / \mathrm{mL})$ & $9.50(6.30 ; 14.54)$ & $9.61(5.33 ; 14.12)$ & \\
\hline
\end{tabular}

Values are expressed as median (25th percentile; 75 th percentile)

Wilcoxon test was calculated to test clinically relevant differences

renal dysfunction is reassuring in terms of CsA safety profile and seems to confirm that renal dysfunction occurs only in predisposed, susceptible subjects. Also positive is the occurrence of hyperkalemia in only one patient. Paresthesia, gingival hyperplasia, and hypertrichosis are well-described CsA side effects, which occurred in our cohorts in a low proportion of patients, from $1.6 \%$ to $5.1 \%$. In terms of TSQM, our patients showed a rather high level of treatment satisfaction, especially in terms of convenience and tolerability, with very little differences among cohorts.

This study suffers from methodological limitations, first of all because its observational design did not allow one to include strictly 


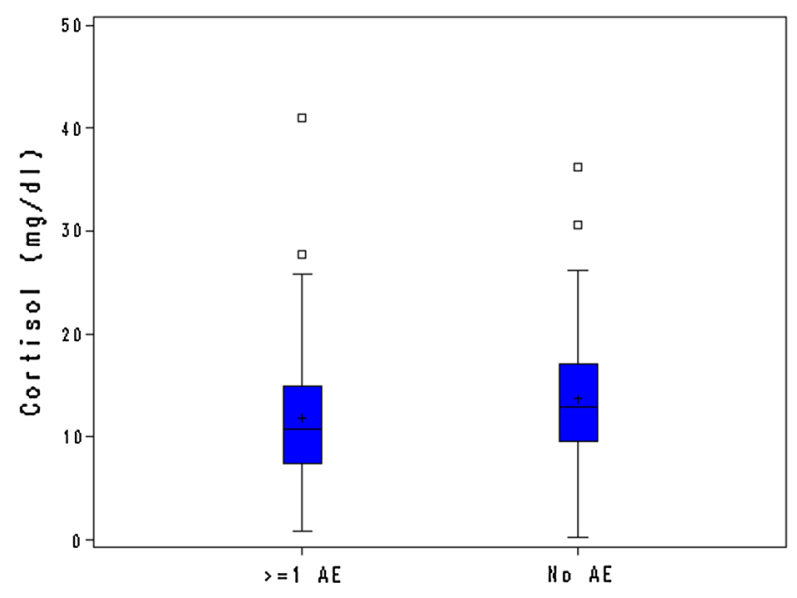

Fig. 3 Cortisol levels in postmenopausal women with and without adverse events. The box plot of cortisol level (mg/ $\mathrm{dL})$ in postmenopausal women who experienced at least one adverse event $(N=66)$ and who did not experience any adverse events $(N=88)$ during the exposure period is shown. The upper and lower edges of the box represent the 75th and the 25th percentile, respectively. The line and the plus symbol inside the box correspond to the median and mean value, respectively. Whiskers are limited by the maximum observation below upper fence (upper limit) and the minimum observation (lower limit). Small squares above the upper edge of the whiskers represent observations beyond the upper fence, defined as 1.5 (interquartile range) above the 75 th percentile

homogeneous patients and treatment regimens. Moreover, study patients were neither newly diagnosed nor naïve to study treatment, thereby by representing a useful example of real-world-based evidence. An important point to be taken into account is that, as a result of the real-world setting in which we conducted the study, we could not control for tobacco, alcohol, or drug use that may interact with CYP3A4 isoenzymes that are involved in CsA metabolism, thus influencing plasma CsA levels. It is known that CSA bioavailability can be influenced by many variables, not only tobacco or specific drugs, so it is not possible to keep them controlled in the study, especially in the real-world setting, where clinicians are free to treat patients according to routine clinical practice [27]. Other possible sources of bias could have been disease and treatment duration, which could have acted as confounders in the association between gender and occurrence of AEs, even though, to partially overcome this bias, IRRs were calculated also after adjusting for such factors. Another possible limitation could be due to the fact that the GENDER study followed patients only up to the end of the ongoing treatment course. The limited follow-up makes our results preliminary and worth being confirmed either. On the other hand, we think that the GENDER ATTENTION study represents a useful example of real-world evidence-based medicine: the observational prospective design allowed us to observe a large cohort of patients treated in a routine clinical practice setting.

\section{CONCLUSIONS}

We believe that the GENDER ATTENTION study may play a role in the context of gender in Italian medicine, being the first study that analyzed sex-specific incidence rate of AEs during CsA treatment of plaque psoriasis in the real life. The results showed a trend towards more side effects in women compared to age-matched men, and age- and hormonal status-related differences in tolerability, with older patients and postmenopausal women reporting a higher incidence of AE. ADRs represent a source of great health concern as well as of increased health and social costs, in terms of hospitalizations, length of hospital stay, loss of productivity, and need to be investigated further and more in depth. This study shed some preliminary light on possible sex- and hormone-related influences on drug tolerability. A better understanding of the impact of gender and hormones on drug responses may enable clinicians to tailor pharmacological treatments to individual subjects or specific classes of patients.

\section{ACKNOWLEDGMENTS}

This study and article processing charges were sponsored by Novartis Farma S.p.A., Italy. Scientific and technical coordination, data management, and statistical support were provided by Medidata s.r.l (Modena, Italy). We thank Renata Perego who provided medical writing 
services on behalf of Novartis Farma S.p.A. All authors had full access to all of the data in this study and take complete responsibility for the integrity of the data and accuracy of the data analysis. All named authors meet the International Committee of Medical Journal Editors (ICMJE) criteria for authorship for this manuscript, take responsibility for the integrity of the work as a whole, and have given final approval for the version to be published.

\section{The GENDER ATTENTION Study} Group. Salvatore Amato (ARNAS Ospedale Civico, Palermo, Italy), Fabio Ayala (Policlinico Universitario Federico II, Napoli, Italy), Ferderico Bardazzi (Ospedale S. Orsola Malpighi, Bologna, Italy), Maria Grazia Bernengo (AOU Città della Salute e della Scienza, Torino, Italy), Maria Rita Bongiorno (Policlinico Paolo Giaccone, Palermo, Italy), Giovanni Borroni (IRCCS Policlinico S. Matteo, Pavia, Italy), Stefano Calvieri (Policlinico Umberto I, Roma, Italy), Piergiacomo Calzavara Pinton (Az. Osp. Spedali Civili, Brescia, Italy), Calcedonio Cannarozzo (Ospedale Garibaldi Centro, Catania, Italy), Serafinella Patrizia Cannavò (Policlinico Universitario G. Martino, Messina, Italy), Angelo Cattaneo (IRCCS Ca' Granda-Ospedale Maggiore Policlinico, Milano, Italy), Rossella Ceschini (Ospedale S. Lucia, Macerata, Italy), Enrico Colombo (Ospedale Maggiore della Carità, Novara, Italy), Maurizio Congedo (Ospedale Vito Fazzi, Lecce, Italy), Francesco Cusano (Ospedale G. Rummo, Benevento, Italy), Ornella De Pità (Istituto Dermopatico dell'Immacolata IRCCS, Roma, Italy), Sergio Di Nuzzo (Ospedale Maggiore, Parma, Italy), Dario Donadio (AORN San Giovanni di Dio e Ruggi di Aragona, Salerno, Italy), Stefano Donelli (Ospedale Guglielmo Da Saliceto, Piacenza, Italy), Raffaele Filotico (Ospedale A. Perrino, Brindisi, Italy), Maria Laura Flori (Policlinico Le Scotte, Siena, Italy), Maurizio Germino (Casa di Cura Città di Udine, Udine, Italy), Giampiero Girolomoni (Ospedale Civile Maggiore-Borgo Trento, Verona, Italy), Franco Kokelj (ASS 1 Triestina, Trieste, Italy), Anna Lanzoni (Ospedale Bellaria, Bologna, Italy), Giuseppe Lembo (Ospedale Sant'Anna, Como, Italy), Andrea Locatelli (A.O. Papa Giovanni XXIII, Bergamo,
Italy), Cristina Magnoni (Az. Osp. Univ. Policlinico, Modena, Italy), Marco Marconi (Ospedale San Paolo, Milano, Italy), Alberico Motolese (Ospedale Macchi, Varese, Italy), Manuela Papini (Ospedale Santa Maria, Terni, Italy), Aurora Parodi (Ospedale Santa Maria, Terni, Italy), Monica Pau (Ospedale Civile S.Giovanni di Dio, Cagliari, Italy), Ketty Peris (Ospedale Civile San Salvatore, L'Aquila, Italy), Stefano Piaserico (Azienda Ospedaliera Padova, Padova, Italy), Angelo Piccirillo (Azienda Ospedaliera San Carlo, Potenza, Italy), Concetta Potenza (Ospedale A. Fiorini, Terracina, Italy), Giuseppe Ricotti (Ospedale Geriatrico INRCA, Ancona, Italy), Patrizio Sedona (Ospedale SS Giovanni e Paolo, Venezia, Italy), Giuseppe Stinco (Azienda Ospedaliero Universitaria, Udine, Italy), Giancarlo Valenti (Ospedale Pugliese-Ciaccio, Catanzaro, Italy), Cleto Veller Fornasa (Ospedale San Bortolo, Vicenza, Italy), Leonardo Zichichi (Ospedale S. Antonio, Trapani, Italy), Alessandra Ori (Ospedale S. Antonio, Trapani, Italy), Christian Amici (Ospedale S. Antonio, Trapani, Italy), Alessandro Zullo (Ospedale S. Antonio, Trapani, Italy), Lucia Simoni (Ospedale S. Antonio, Trapani, Italy), Nicole Lanci (Ospedale S. Antonio, Trapani, Italy), Chiara Longagnani (Ospedale S. Antonio, Trapani, Italy), Saide Sala (Ospedale S. Antonio, Trapani, Italy), Francesca Trevisan (Ospedale S. Antonio, Trapani, Italy), Giovanna Magri (Ospedale S. Antonio, Trapani, Italy).

Disclosures. Delia Colombo is a part-time employee of Novartis Farma Italy and received grants from Allergan and Aventis. Nicoletta Cassano has been a speaker and/or a scientific consultant for Astellas, Abbvie, Leo Pharma, Merck-Serono, Merck Sharp \& Dohme, Novartis, Pfizer, Rottapharm madaus, and UCB Pharma. Alessandra Graziottin has been a speaker at Bayer, Deakos, Fidia, Italfarmaco, Lolipharm, Menarini, Shionogi, Specchiasol, and Zambon; a member of the advisory board at Bayer, Fidia, and Menarini; a consultant at Alfa Wassermann, Bayer, Deakos, Epitech, Fidia, Italfarmaco, Lolipharm, Menarini, Shionogi, and Zambon. Gino Antonio Vena has been a speaker and/or a scientific consultant for Astellas, Abbvie, Leo Pharma, Merck-Serono, Merck Sharp \& 
Dohme, Novartis, Pfizer, Rottapharm madaus, and UCB Pharma. Giovanni Gualberto Fiori is an employee of MediNeos, Italy, and the CRO in charge of conducting the GENDER ATTENTION study (which at that time was called MediData srl). Emanuela Zagni is a full-time employee of Novartis. Sergio Chimenti has received advisory/speaker honoraria and/or research funding from Abbvie, MSD, Novartis, and Pfizer. Clara De Simone has received advisory/speaker honoraria and/or research funding from Pfizer, Abbvie, Janssen, Celgene, Leopharma, Novartis. Gilberto Bellia was employed by Novartis during study execution and manuscript elaboration while now he is employed by UCB. Giuseppe Banfi, Luca Stingeni, Enzo Berardesca, Giuseppe Micali, and Giuseppe Albertini declare that they have no competing interests.

Author contributions. Delia Colombo was responsible for project conception and original idea. Delia Colombo, Giovanni Gualberto Fiori, Giuseppe Banfi, Nicoletta Cassano, Alessandra Graziottin, and Gino Antonio Vena collaborated in the study design and development. Delia Colombo, Emanuela Zagni, and Gilberto Bellia collaborated in medical writing. Nicoletta Cassano, Giuseppe Banfi, Gino Antonio Vena, Luca Stingeni, Sergio Chimenti, Enzo Berardesca, Giuseppe Micali, Giuseppe Albertini, and Clara De Simone made substantial contributions to the acquisition of data for the work. All authors contributed to critically reviewing the manuscript. The GENDER ATTENTION study group made substantial contributions to the acquisition of data for the work and to conducting the study.

Compliance with ethics guidelines. All subjects gave their informed consent for participation in the study. The study protocol was reviewed by the independent ethics committee (IEC) or institutional review board (IRB) of each center. The study was carried out in accordance with the approved applicable guidelines.

Data availability. The datasets generated during and/or analyzed during the current study are not publicly available because they are property of Novartis Farma S.p.A., but they are available from the corresponding author on reasonable request.

Open Access This article is distributed under the terms of the Creative Commons Attribution-NonCommercial 4.0 International License (http://creativecommons.org/licenses/by-nc/4.

$0 /$ ), which permits any noncommercial use, distribution, and reproduction in any medium, provided you give appropriate credit to the original author(s) and the source, provide a link to the Creative Commons license, and indicate if changes were made.

\section{REFERENCES}

1. Pirmohamed M, James S, Meakin S, et al. Adverse drug reactions as cause of admission to hospital: prospective analysis of 18,820 patients. BMJ. 2004;329(7456):15-9.

2. Patel H, Bell D, Molokhia M, et al. Trends in hospital admissions for adverse drug reactions in England: analysis of national hospital episode statistics 1998-2005. BMC Clin Pharmacol. 2007;7:9.

3. Franconi F, Carru C, Spoletini I, et al. A GENS-based approach to cardiovascular pharmacology: impact on metabolism, pharmacokinetics and pharmacodynamics. Ther Deliv. 2011;2(11):1437-53.

4. Fattinger K, Roos M, Vergeres P, Holenstein C, Kind $B$, Masche $U$, et al. Epidemiology of drug exposure and adverse drug reactions in two Swiss departments of internal medicine. Br J Clin Pharmacol. 2000;49(2):158-67.

5. Franconi F, Campesi I, Occhioni S, Antonini P, Murphy MF. Sex and gender in adverse drug events, addiction, and placebo. Handb Exp Pharmacol. 2012;214:107-26.

6. Women's Health. GAO report to Congressional Requesters 01-754. 2001.

7. Zender R, Olshansky E. Women's mental health: depression and anxiety. Nurs Clin $\mathrm{N}$ Am. 2009;44(3):355-64.

8. Sikdar KC, Alaghehbandan R, MacDonald D, et al. Adverse drug events in adult patients leading to emergency department visits. Ann Pharmacother. 2010;44(4):641-9. 
9. Anthony M. Male/female differences in pharmacology: safety issues with QT-prolonging drugs. J Women Health. 2005;14(1):47-52.

10. Parisi R, Symmons DP, Griffiths CE, Ashcroft DM, Identification and Management of Psoriasis and Associated Comorbidity (IMPACT) project team. Global epidemiology of psoriasis: a systematic review of incidence and prevalence. J Invest Dermatol. 2013;133(2):377-85.

11. Atkinson MJ, Sinha A, Hass SL, et al. Validation of a general measure of treatment satisfaction, the Treatment Satisfaction Questionnaire for Medication (TSQM), using a national panel study of chronic disease. Health Qual Life Outcomes. 2004;2:12.

12. Sandimmun Neoral (and associated names), summary of product characteristics. 2015. http://www. ema.europa.eu/docs/en_GB/document_library/Refer rals_document/Sandimmun_Neoral_30/WC5001448 86.pdf. Accessed 1 Jan 2017.

13. Franconi F, Brunelleschi S, Steardo L, Cuomo V. Gender differences in drug responses. Pharmacol Res. 2007;55(2):81-95.

14. Martin RM, Biswas PN, Freemantle SN, Pearce GL, Mann RD. Age and sex distribution of suspected adverse drug reactions to newly marketed drugs in general practice in England: analysis of 48 cohort studies. Br J Clin Pharmacol. 1988;46(5):505-11.

15. Domecq C, Naranjo CA, Ruiz I, Busto U. Sex-related variations in the frequency and characteristics of adverse drug reactions. Int J Clin Pharmacol Ther Toxicol. 1980;18(8):362-6.

16. Tran C, Knowles SR, Liu BA, Shear NH. Gender differences in adverse drug reactions. J Clin Pharmacol. 1988;38(11):1003-9.

17. Caamano F, Pedone C, Zuccala G, Carbonin P. Sociodemographic factors related to the prevalence of adverse drug reaction at hospital admission in an elderly population. Arch Gerontol Geriatr. 2005;40(1):45-52.

18. Camargo AL, Cardoso Ferreira MB, Heineck I. Adverse drug reactions: a cohort study in internal medicine units at a university hospital. Eur J Clin Pharmacol. 2006;62(2):143-9.

19. Light KP, Lovell AT, Butt H, Fauvel NJ, Holdcroft A. Adverse effects of neuromuscular blocking agents based on yellow card reporting in the UK: are there differences between males and females? Pharmacoepidemiol Drug Saf. 2006;15(3):151-60.

20. Onder G, Pedone C, Landi F, et al. Adverse drug reactions as cause of hospital admissions: results from the Italian Group of Pharmacoepidemiology in the Elderly (GIFA). J Am Geriatr Soc. 2002;50(12):1962-8.

21. van den Bemt PM, Egberts AC, Lenderink AW, et al. Risk factors for the development of adverse drug events in hospitalized patients. Pharm World Sci. 2000;22(2):62-6.

22. Trifirò G, Calogero G, Ippolito FM, et al. Adverse drug events in emergency department population: a prospective Italian study. Pharmacoepidemiol Drug Saf. 2005;14(5):333-40.

23. Kashuba AD, Nafziger AN. Physiological changes during the menstrual cycle and their effects on the pharmacokinetics and pharmacodynamics of drugs. Clin Pharmacokinet. 1988;34(3):203-18.

24. Klein SL. Sex influences immune responses to viruses, and efficacy of prophylaxis and therapeutic treatments for viral diseases. Bioassays. 2012;34(12):1050-9.

25. Villa E, Karampatou A, Cammà C, et al. Early menopause is associated with lack of response to antiviral therapy in women with chronic hepatitis C. Gastroenterology. 2011;140(3):818-29.

26. Colombo D, Chimenti S, Giannetti A, et al. Therapeutic approaches in psoriasis: a post hoc analysis of the PSYCHAE study from a gender point of view. Clin Dermatol. 2013;2(2):77-83.

27. Colombo D, Lunardon L, Bellia G. Cyclosporine and herbal supplement interactions. J Toxicol. 2014;2014:145325. 